\title{
Can Authority be Sustained while Balancing Accessibility and Formality?
}

\begin{abstract}
Economics has developed into a quantitative discipline that makes extensive use of mathematical and statistical concepts. When writing a dictionary for economics undergraduates it has to be recognised that many users will not have sufficient training in mathematics to benefit from formal definitions of mathematical and statistical concepts. In fact, it is more than likely that the user will want the dictionary to provide an accessible version of a definition that avoids mathematical notation. Providing a verbal description of a mathematical concept has the risk that the outcome is both verbose (compared to a definition using appropriate mathematical symbols) and imprecise. For the author of a dictionary this raises the question of how to resolve this conflict between accessibility and formal correctness. We use a range of examples from the Oxford Dictionary of Economics to illustrate this conflict and to assess the extent to which a non-formal definition can be viewed as authoritative.
\end{abstract}

\section{Introduction}

The paper is based on our experiences of preparing the revised edition of the Oxford Dictionary of Economics and especially with delivering appropriate formality and rigor while ensuring that the dictionary provided definitions that were accessible by its intended audience. It was determined at the start of the revision process that the primary audience would be first-year undergraduate students in economics. We also aimed to address much of the material to students of economics at a lower level and specifically included technical definitions for more advanced students. Although they were not our primary audience we also expected that much of the content would be beneficial to non-specialist readers. In addressing the dictionary to this audience the major challenge was to select the level of technical formality. If this was too low then the dictionary would merely describe but not explain. If it was too high then the dictionary would not be accessible for its target audience. In the discussion that follows we explain why the level of technical formality is so important in an economics dictionary and describe our approach to resolving this difficulty.

The $4^{\text {th }}$ edition of the Oxford University Press Dictionary of Economics (referred to from here forward as the Dictionary) was published in 2012. This edition was a revision by Hashimzade and Myles of the $3^{\text {rd }}$ edition that included many concepts that had come to prominence during the financial crisis of 2009. The $3^{\text {rd }}$ edition, published in 2009, was a major revision by Hashimzade and Myles of the $2^{\text {nd }}$ edition written solely by John Black and published in 2002. In our discussion we will focus upon the production of the $3^{\text {rd }}$ edition because that is the point at which the new style was imposed upon the dictionary. The intention of the publishers when commissioning the 3rd edition was that there would be some minor updating of the content given economic and, perhaps, political developments, as well as the addition of a small number of new entries reflecting the development of economics as a discipline. This was also our original intention until the point at which we carefully read and evaluated the content of the $2^{\text {nd }}$ edition.

There were several other dictionaries available at the time of preparing the 3 rd edition. These were very carefully analysed for style and a thorough comparison made of content. A spreadsheet

\begin{tabular}{ll}
\hline * Nigar Hashimzade & Georgina A. Myles \\
Department of Economics & Department of Economics \\
Durham University & University of Essex \\
Durham,DH1 3LB & Colchester, CO4 3SQ \\
UK & UK \\
nigar.hashimzade@durham.ac.uk & gamyles@essex.ac.uk
\end{tabular}

Gareth D. Myles

Department of Economics

University of Exeter

Exeter, EX4 4PU

$U K$

gdmyles@ex.ac.uk 
was compiled of the significant entries in these alternatives that were not in the 2 nd edition. The analysis of the alternative dictionaries revealed a range of problems. First, there was a general tendency for these dictionaries to be out-of-date with respect to recent advances in the discipline. Second, the 2nd edition and all the alternatives were incomplete and in each various important topics were absent. Finally, the level of mathematical formality was too low in all of these dictionaries. We discuss this important issue in considerable detail below. A side issue: with increasing use of the internet online definitions had become readily available and we needed to add value over these.

The revisions for the 3rd edition focussed upon changing the style of many existing entries, removing value judgements, expressions of opinion, and political bias. We also eliminated the considerable repetition in entries (see later examples). The $2^{\text {nd }}$ edition included entries on basic mathematics, such as linear equation, that were removed. This decision was based on the view that it was an economics dictionary and not a mathematics dictionary. We also had to amend substantially many economic entries that had mathematical content. Numerous definitions were added on econometric concepts, this being one area that had transformed since the 2 nd edition.

The first set of changes primarily reflected the individual academic styles of the new co-authors and their assessment of the extent to which any personal and/or societal value judgements, and political views, should be included within academic writing. The changes reflect the view that no such expressions should be included. The revised definitions consequently focus on concepts and facts, and implications derivable by logical argument. It is the second group of changes concerning mathematics and econometrics that are the focus of this paper. These changes reflect the transformation of economics as a discipline and the transformation of education in economics. It is here that the issue of achieving the appropriate level of formality while retaining accessibility had to be confronted.

The final questions that we address in the paper are whether a paper dictionary is justified and what an online version of our dictionary could deliver. There are many existing online dictionaries, and so it might be argued that the marketplace is crowded and a new entrant is unnecessary. However, a review of online dictionaries will show that definitions are often too brief and superficial, and that economic concepts are often dominated in context by financial applications. There is also the issue of reliability that arises with the anonymous nature of online definitions.

Section 2 reviews the increase in formality in economics from a historical perspective via some landmark contributions. Section 3 discusses the role of formal reasoning in economics. Section 4 describes how we tried to meet the conflicting requirements of accessibility and formality using examples from the Dictionary. Section 5 explores authority and accessibility, and again uses example from the Dictionary. Section 6 considers what an online dictionary could contribute. Conclusions are given in section 7.

\section{Formality in economics}

Over the past century economics has evolved rapidly as an academic discipline. It has developed from literary roots in political economy to a highly analytical science that uses advanced mathematics and statistics. Education in economics reflects this transformation. Even in the very recent past the emphasis of education was upon debate and discussion and focussed around the "essay". It has now become increasingly concerned with the teaching of analytical techniques and assessment in the application of those techniques.

These changes have been matched by an evolution in the form of communication in economics. The political economy of the 19th century emerged as a contribution to political debate and was expressed in standard vocabulary with limited use of technical terms. This situation began to change at the start of the 20th century when economics began to develop as a scientific discipline in its own right. It has now become a mathematical and formal discipline that places great importance on analytical and logical rigor. We now describe some of the landmarks in this evo- 
lution to provide a context for the issues that need to be confronted when writing a dictionary of economics.

Two notable landmarks were the Economic Journal papers by Frank P. Ramsey, a British economist, on economic growth and optimal taxation. The central component of both papers was a substantive mathematical analysis as opposed to a discussion of institutions or data. Ramsey (1927) analysed the optimal set of commodity taxes by solving the maximisation of social welfare subject to the government budget constraint, elegantly expressing the necessary conditions in terms of elasticities (ratios of proportional changes in prices and quantities). The Ramsey (1928) growth paper used the principles of control theory to characterise the optimal consumption path over an infinite lifecycle. At the time of publication the papers stood in marked contrast to the other research published in the same issues of the journal. For example, "A Mathematical Theory of Saving" by Ramsey in vol. 38 of the Economic Journal was preceded by "Increasing Returns and Economic Progress" by A.A. Young and followed by "The Incidence of Taxation in Agriculture" by J.A. Venn; neither of these presented a formal mathematical treatment of the issue in the title. Typically for the contemporary economic literature, both these papers are purely descriptive, although a "Note" to Young's paper contains a schematic diagram to illustrate the main point.

The 1939 treatise Value and Capital by John Hicks, another outstanding British economist, introduced matrix methods into the mainstream. The significance of Value and Capital was that it was intended to be widely read by economists in general, and not just by a narrow audience of specialists. The matrix methods of Hicks eventually became a staple component of economics until being displaced by more general and more rigorous alternatives.

The Theory of Games (1947) by John von Neumann and Oscar Morgenstern demonstrated beyond doubt the value of formal reasoning. It is no exaggeration to say that the treatise created an entirely new academic discipline of game theory and, simultaneously, established some of the most profound results in that discipline. The formalisation of game theory completely changed the direction of economic reasoning and showed that the subject was a suitable field for applied mathematics.

The benefit of further abstraction was shown by Kenneth Arrow's Social Choice and Individual Values (1951). The book took as its starting point a disparate collection of observations about voting processes but finished with one elegant conclusion, now known as Arrow's Impossibility Theorem, which proved the general principle underlying the individual results. The argument used by Arrow was not to add further cases to the existing set but to start from an entirely new perspective. A set of axioms were proposed and the logical consequences of those axioms derived. The emergence of the Impossibility Theorem was a resounding success for formality.

Finally, Theory of Value (1959) by Gerard Debreu completed a programme of research begun in the 19th century. Economists model markets by thinking of demand and supply being determined by prices. An equilibrium occurs when demand is equal to supply (see the discussion below for more analysis of this point). The natural question to ask is whether there exists a set of prices that ensure the markets for all goods can be in equilibrium simultaneously. Many great economists, including Adam Smith, Leon Walras and Abraham Wald, had contributed to the discussion of the existence of equilibrium in a competitive market but none had resolved the question. The first proofs of existence appeared in 1954 (Arrow/Debreu, McKenzie) and drew on mathematical analysis and the theory of convexity to provide formal conditions under which a competitive equilibrium would exist. Theory of Value provided the defining statement of this literature using the most general conditions known at the time. The imposing volume Microeconomic Theory (1995) by Mas-Collel, Whinston and Greene, now a mandatory textbook for doctoral students in economics worldwide, is effectively a final compendium of this literature.

These advances in the mathematical formalisation of economic models were paralleled by the development of the methods for quantitative analysis of economic data. Early attempts to establish quantitative relationships between economic and political variables can be traced to the 16th century in work by "political arithmeticians". In the late 19th century economists started apply- 
ing the new achievements of probability theory and statistics to the analysis of patterns in various economic, social, and demographic indicators.

In the first decades of the 20th century much of the interest was in quantifying the patterns of "economic cycles", without, however, detailed analysis of the forces driving the cycles. However, it was not until the 1930-40s that statistical methods came into systematic use in the quantitative analysis of economic relationships implied by economic models, and econometrics became an acknowledged discipline. The focus was on adapting the existing statistical methods and developing new methods given the specific features of economic data (such as, for example, the fundamental inability of a researcher to control external factors, as one would do in an experiment in physics or chemistry, or to observe and measure important factors governing economic decisions, such as individual tastes or degree of impatience).

In the 1960s the main focus was on macroeconomic forecasting, mostly based on ad hoc "intuitive" assumptions on the links between aggregate economic indicators; with the development of formal economic models new econometric techniques emerged that could be used to test more sophisticated models. This progress was greatly helped by the increase in the available computing power. Modern econometrics is, effectively, a branch of applied mathematics, and even the very basic knowledge of econometrics requires formal mathematical training.

Thus, mathematical background and analytical rigor are nowadays, perhaps, the most valuable assets of a successful economist. Rankings of journals reflects this. In the influential Diamond List and Keele Ranking most of the highest-ranked journals are the most analytical. For our narrative it is important to note that the research literature was far ahead of economics education. This was a consequence of the fact that many of the contributors were trained as mathematicians or physicists. So they naturally used the analytical methods of those disciplines. The teaching of formal methods did not really enter into the economics curriculum until the 1970s, and then to greatly varying degrees in different institutions.

By the end of the 20th century economics was in a position of a discipline in which research was far advanced over education. During the transition many students who received an education in economics did not receive an adequate training in mathematical and statistical techniques. There is a gap that has to be spanned by the author of a dictionary, who, therefore, must have a comprehension of the formal methods and an ability to convey meaning to an audience that has not.

\section{The role of formal reasoning}

The brief history in section 2 traced the development of formal methods in economics. Many people are surprised when they first discover the economics is primarily a mathematical and statistical discipline. Perhaps this is because most exposure for the non-specialist is via the media where basic economic statistics are discussed and elementary ideas are enrolled into political debate. If a non-formal version of economics is suitable for these purposes, why has academic analysis developed economics into a formal discipline?

The first reason is the standard applied to all disciplines: specialists need a language in which they can communicate effectively without the looseness and vagaries of everyday communication. This argument only goes part of the way since it justifies a formal language but not necessarily mathematics as the basis of that language. The standard additional argument is that the use of mathematics ensures that arguments are logically correct and coherent. From this perspective, the need to formalise an argument prevents any doubt about what has been assumed and what is subsequently established. It also provides a basis from which assumptions can be tested, and permits their effect upon the outcome of the argument to be tested. These arguments could be applied equally to any of the social sciences yet economics has followed a different path to the others. The reason for this must lie in the fact that economics is fundamentally quantitative in the objects that it discusses. Economic indicators, such as output, consumption, inflation, exchange 
rates, prices, and unemployment, are all measurable quantities, and so immediately invite the application of mathematics.

From an educational perspective the formal aspect of economics is of considerable value to students. The completion of an economics degree is a signal of analytical competency and numeracy. These are valued capabilities in the labour market and are reflected in the salaries paid to graduate economists. The formality also makes life easier for academic staff since the teaching and examining of mathematical exercises is less time consuming than the grading of essays. This is a particular benefit when the student to staff ratio is high (due to increasing demand for economics degrees), or when teaching is in a non-native language either for students or for the instructor (due to growing internationalisation of economics departments).

There are objectors to this status quo who argue that the formal approach to economics has not delivered. There has been a recent proposal in the UK to move to a less formal curriculum, but this is unlikely ever to be taken seriously since it reflects the views of a minority who have not succeeded in formal economics and therefore are not perceived by the majority to have credibility in this argument. Another objection is that there are very few substantive results in some key areas of economics. For example, the major conclusion of consumer theory is that the Slutsky substitution matrix is negative semi-definite. This is a weak mathematical restriction but has neither been validated or rejected by empirical research. This final point can be developed further into the observation that none of the formal results has been verified by either data or experiment. The argument has also been advanced that simpler reasoning is more successful than sophisticated reasoning. An example of this is the view of some macroeconomists that the simple IS-LM model provides as good an explanation of the macro economy as any of the more sophisticated models. The perceived failure of complex models to predict the recent financial crisis is another argument against their use.

We cannot agree with these arguments. The proponents of the IS-LM model also failed to predict the financial crisis, and it could be argued that the financial crisis was partly caused by a deficit of formal training of participants in financial markets. If the purchasers of collateralised securities had had the proper training to evaluate what they were purchasing then the inherent riskiness of the instruments could have been correctly assessed. In addition, some of the results of formal economics have completely transformed the world. The most notable example is the BlackScholes analysis of options pricing which has underpinned the flourishing of financial markets in the past 30 years. On a slightly smaller scale, the application of auction theory raised billions of pounds for the UK government in the $3 \mathrm{G}$ auction. Game theory has also featured prominently in the planning of warfare.

In addition, even if formality does not deliver in the present then it is a step to a proper economics for the future. The subject is still in its infancy and will need the techniques for the future. There is also an issue of applicability of existing techniques from natural sciences in the economics context. Even a small step from a simplified to a more "realistic" model often greatly increases complexity (all electrons are the same whereas all people are different). Techniques for the analysis of complex systems are relatively new in mathematics. Also, development of computing power makes at least some numerical analysis of complex systems possible; hence, programming becomes another useful formal skill.

Whichever of these arguments is correct it is unlikely that formal reasoning in economics will become redundant in the near future. If anything, a greater emphasis will be placed on formality in the short term due to the rewards (appointments, publications, market value) for more formal work. In the words of game theory, academic economists are engaged in a form of "rat race" in which the user of the greater degree of formality receives a greater prize, prompting others to strive for an even greater degree of formality in a constant competition for prizes. The discipline rewards technical expertise, and so encourages ever greater technical sophistication.

The implication of the use of formality in economics is that it requires specialist knowledge to provide the translation of the formal language of the discipline into a form of language that can 
be understood by the target audience of a dictionary. It cannot be done by collecting together the definitions that are publicly available online since these are not always correct (and an example is given below). Instead, it can only be done by specialist compilers who understand the formality and appreciate the requirements of the target audience. We also wish to argue that it is probably a task that requires more than one specialist compiler given the current breadth of the discipline. These compilers also need to have complementary expertise.

\title{
4. Defining concepts
}

The construction of a definition of a concept for a dictionary of economics faces the compiler with two major challenges. First, the compiler of a dictionary may not have had the training necessary to understand many formal concepts. Second, a dictionary needs to explain ideas that are beyond the training of many readers. The first point can result in errors, misunderstandings, and omissions, of which of a typical reader will be unaware. With the readers relying on the authority of the compiler of the dictionary, there is a danger that an erroneous definition becomes an accepted wisdom. The second point provides the challenge for the compiler.

The 1st and 2nd editions of the Dictionary were written by an Oxford-educated author John Black, a well-known British economist and author of many books and journal articles. Paradoxically, in economics Oxford took the best students but provided the worst training. It did not recognise the advance in technique, and so the economics programme included no mathematics or statistics until the late 1980s. The Oxford tradition in economics put more emphasis on the ability to understand and express economic concepts and relationships at an intuitive level, rather than in the form of mathematical relationships. Such training did not provide the best preparation for a rapidly advancing and increasingly mathematical discipline.

The early editions of the Dictionary were a product of this tradition. The number of entries that intended to give accessible definitions of advanced concepts was very low. Many important results to be found in the technical literature were absent, even when they involved the most fundamental concepts. Revising the definitions in the Dictionary according to the modern approach highlighted the challenge of achieving accessibility while maintaining adequate formality.

We now use several examples to illustrate these points. We demonstrate the weaknesses of the definitions used in the $2^{\text {nd }}$ edition and describe how we attempted to make improvements.

\subsection{Example 1}

The first example is consumer behaviour. The definition from the $2^{\text {nd }}$ edition is:

\begin{abstract}
consumer behaviour The way in which consumers choose how to spend their incomes. One theory of consumer behaviour views consumers as having *utility functions showing the levels of satisfaction they will derive from every possible set of goods and services. They choose their expenditures to maximize their utility subject to the constraints imposed by their incomes and the prices facing them. This view assumes that tastes are given, independent, and fully known, and that information is free, complete, and reliable. Critics of this position point out that the set of available goods and services is continually changing, that knowledge about what is available is partial, expensive, and unreliable, and that consumers' own tastes evolve as they age and their marital status changes. It should also be pointed out that a large proportion of consumers are members of multi-person households, and are attempting in their spending to please more than one person. Consumers thus work partly on a basis of *satisficing, that is, repeating satisfactory purchases until something goes wrong, and partly on a basis of trial and error, to explore their own reactions to products they have not previously tried. This position leaves more scope for *advertising to influence purchases than the view that consumers maximize a fixed utility function subject to known constraints.
\end{abstract}

The first italicised comment is formally incorrect. The theory does not view consumers as having utility functions. Instead, it provides conditions under which a consumer will behave as if their choices are guided by the maximisation of a utility function. The second italicised statement that "information is free, complete, and reliable" dismisses the entire theory of consumer choice un- 
der uncertainty, or with costly information acquisition. The invocation of "critics" fails to identify who these critics may be, or to provide direction as to where these arguments can be found. The statement about multi-person households raises an important point but does not develop it adequately. Once this point is raised the reader needs to be directed to an entry on household decision making to explore the implication. The final two italicised phrases are an example of an entirely speculative statement that has no evidential basis.

The revised version given in the $3^{\text {rd }}$ edition abbreviates the definition to maintain the core information. It provides the correct statement on the utility function and briefly explores the implications of satisficing:

\begin{abstract}
consumer behaviour The way in which consumers choose how to spend their incomes. In economic theory it is usually assumed that every consumer is aware of their wants and how to satisfy them and that consumers attempt to maximize the benefits received from consumption of goods and services. Thus, consumers behave as to maximize their preferences or *utility function. An alternative view is that consumers work partly on a basis of *satisficing, that is, repeating satisfactory purchases until something goes wrong, and partly on a basis of trial and error, to explore their own reactions to products they have not previously tried. This position leaves more scope for *advertising to influence purchases than the view that consumers maximize a fixed utility function subject to known constraints.
\end{abstract}

\title{
4.2. Example 2
}

The next example uses two related statistical terms. The point of this example is to show how the lack of organisation of the definitions and the repetition that was involved can undermine their value for the reader. The entry for the first term, heteroskedasticity (the 2 nd edition uses an alternative spelling, with $\mathrm{c}$ in place of $\mathrm{k}$ ), provides a definition in the first two sentences, then mentions the second term, homoskedasticity, and after that returns to heteroskedasticity. The entry for homoskedasticity is even less consistent: the first two sentences define homoskedasticity, the third sentence refers to heteroskedasticity, the fourth returns to homoskedasticity, and the fifth switches again to heteroskedasticity. The final sentence is the same in both entries, even though it is less relevant for homoskedasticity. We use italics to highlight these sentences.

The definitions from the 2 nd edition:

heteroscedasticity Having different variances. Data are heteroscedastic if their variations are not consistent with being random drawings from the same population. This is contrasted with *homoscedasticity, where the data appear consistent with being random drawings from the same distribution. Many statistical procedures are not strictly valid for heteroscedastic data. Data on time-series drawn from a world with changing structures, or cross-sections of different industries or countries, can easily turn out to be heteroscedastic.

\begin{abstract}
homoscedasticity Having the same variance. Data are homoscedastic if their variations are consistent with being random drawings from the same population. This is contrasted with *heteroscedasticity, that is, having different variances. Many statistical procedures are only strictly valid for homoscedastic data. Data on time-series drawn from a world with changing structures, or cross-sections of different industries -or countries, can easily turn out to be heteroscedastic.
\end{abstract}

The revisions removed the repetition and ordered material in a more consistent way. We also tried to explain the relevance of concepts and to link the headword to related concepts. These are the revised definitions:

heteroskedasticity Having different variances. A set, or a vector of observations is heteroskedastic if the variance is different for different observations. Heteroskedasticity observed in the cross-sectional data typically is related to the scale effect: often, larger cross-sectional units are subject to larger erratic values of the disturbance. In time series data it may take the form of serial correlation in the variance (*autoregressive conditional heteroskedasticity, or ARCH). It may also be introduced by model misspecification. In the presence of heteroskedasticity OLS estimators of the coefficients are consistent but inefficient; those of the standard errors are inconsistent, and hence the standard inference based on the estimated standard errors is invalid. Among the popular tests for heteroskedasticity are *BreuschPagan, *Glejser, and *White's tests. Two approaches to estimation with heteroskedastic data are the 
*generalized least squares (both the coefficients and the standard errors are re-estimated) and the het-
eroskedasticity-consistent standard errors (only the estimated standard errors are corrected).

homoskedasticity Having the same variance. A set, or a vector, of observations is homoskedastic if the variance is the same for all observations. This is one of the classical linear regression assumptions underlying the *Gauss-Markov theorem. Violation of this assumption is called *heteroskedasticity.

\title{
4.3. Example 3
}

Here is the definition of an equilibrium (perhaps, the central concept in economics) in the 2nd edition. We have highlighted the key words using italics:

equilibrium A situation in which nobody has any immediate reason to change their actions, so that the status quo can continue, at least temporarily. This concept is applied in economics in a number of related ways. These concern microeconomics, macroeconomics, and game theory.

What is wrong with this? "Nobody" suggests people - but a definition should encompas firms and governments. The use of "immediate reason" suggests the existence of time, but we want the definition to apply to both temporal and atemporal settings. The same comment applies to "continue". Finally, the use of "related" is simply too vague.

This is the entry for equilibrium in the $3^{\text {rd }}$ edition:

equilibrium An equilibrium can be defined either as a position of balance in the economy or, equivalently, as a situation in which no agent in the economy has any incentive to modify their chosen strategy. The first definition is derived from the perspective of equilibrium occurring when the forces of supply are balanced by the forces of demand. The second definition derives from the theory of games and is illustrated by the equilibrium of an oligopolistic market in which all firms are satisfied with their choice of output level given the choices of their rivals.

\subsection{Example 4}

Further difficulties are illustrated by the definition of duality which has proved an important concept in the analysis of the consumer and the firm. It has also found numerous application in applied fields of economics.

From the $2^{\text {nd }}$ edition of the Dictionary, with key phrases highlighted with italics:

\begin{abstract}
duality The fact that economic problems stated in terms of one set of variables can also be considered in terms of an alternative set. For example, in *linear programming, the problem may be to maximize the value of output subject to resource constraints. The dual problem corresponding to this involves choosing shadow prices to minimize the value put on resources, consistent with firms breaking even producing outputs subject to constraints imposed by the available techniques of production. It can be shown that these approaches lead to the same results. Which problem is simpler to handle computationally varies from case to case.
\end{abstract}

This definition is wrong in two significant ways. First, it is not one set of variables and an alternative set - it is the same sect of choice variable for both versions of the problem. Second, the dual problem described is simply wrong. It is, in fact, the choice of inputs to minimise resource cost subject to achieving the required output. Finally, the last sentence is a throwaway remark that has no basis in evidence.

The revised definition in the $3^{\text {rd }}$ edition of the Dictionary states that:

duality The idea that there are multiple ways of viewing a single issue. More formally, duality applied to optimization theory states that every maximization problem has a dual minimization problem and vice versa. Any problem can be transformed into its dual by interchanging constraints and objectives. In consumer theory the problem of maximizing utility subject to a budget constraint is dual to minimizing expenditure subject to achieving a given level of utility. The use of duality provides alternative ways to represent the solution. For example, the *expenditure function and the *indirect utility function are equivalent representations of consumer behaviour. 
In this section we have illustrated our approach to the revision of the definitions and some of the issues that had to be addressed. We now move on to the important issue of combining correctness with accessibility.

\section{Authority and accessibility}

In preparing the $3^{\text {rd }}$ edition it was always our intention that the Dictionary should be accepted as authoritative. We view authority as being achieved if a dictionary is accepted as a standard source of reference with definitions that are unanimously approved. The latter may be an unattainable ideal given the proximity of some aspects of economics to political dogma, but it is a commendable aspiration. Our intention for correctness had to be pursued within the constraints imposed by the needs of the target audience.

As a discipline, economics admires technical sophistication so that there would, by necessity, be a divergence between a dictionary and the academic literature. It is most likely that many of the target audience will be looking to the Dictionary to provide an accessible explanation of a formal term. We could not provide the formal definitions at the level of the reputable research journals because to do so would not serve the target audience. In fact, it would be next to impossible to serve our target audience of undergraduates while simultaneously reaching out to advanced graduate students.

Thus, a definition provided by an authoritative source must be credible and widely accepted. To a considerable extent, the authoritativeness is signalled by the stamp of a publisher, and the academic reputation of Oxford University Press is beyond any doubt one of the highest in the world. This, however, is not enough. Credibility and acceptance can be corroborated by the fact that the same definition is provided by many different sources, suggesting unanimity or, at least, a majority being in agreement. However, many internet definitions appear to be copied from each other and often repeat the same mistake.

\subsection{Example 5}

An interesting definition to illustrate these observations is that of a corner solution. Searching on internet gives the typical definition:

\footnotetext{
A corner solution is a special solution to an agent's maximization problem in which the quantity of one of the arguments in the maximized function is zero. The more usual solution will lie in the non-zero interior at the point of tangency between the objective function and the constraint. ... (www.wordvia. $\underline{\text { com/dictionary/corner solution) }}$
}

The internet definition of a corner solution is similar to the definition that is found in the $2^{\text {nd }}$ edition:

corner solution A solution to a system of equations where some variables are zero....

As a general definition of a corner solution both of these are incorrect. They can be correct if provided with an appropriate context but, in the absence of such context, the definitions are incorrect and misleading.

To see why the definitions are incorrect consider the problem of maximising the value of the function $-x^{2}$ on the interval $[-1,1]$. A graph of the function is shown in Figure 1, and it is clear that the value that gives the maximum is $x=0$. This is an interior point of the interval on which the function is defined. A corner solution in this case would be either at -1 or at 1 . This shows how the definition can be incorrect in the absence of further elaboration of the situation it is describing even though it does give the impression of authority. 


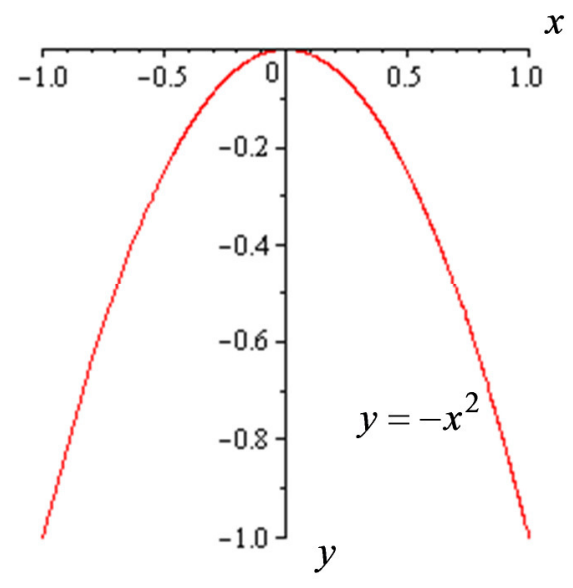

Figure 1. The graph of $-x^{2}$

It might be argued that the additional context is not necessary because that is provided by the fact that the definition is located within a dictionary of economics. A further economic example can show why this argument does not apply, and that there are very standard examples of economic analysis for which the definitions are incorrect.

Most undergraduate students of economics will be taken through an analysis of the saving decision of a consumer. The usual setting for this problem is to assume there are two time periods, say, young age and old age. The consumer has an income in both periods, and in the first period must choose how much to save. The underlying issue is the allocation of consumption across the two periods and, given the incomes, it is the level of saving that determines this. The choice variable of the consumer is the level of saving, $s$. Three categories of outcome are then possible:

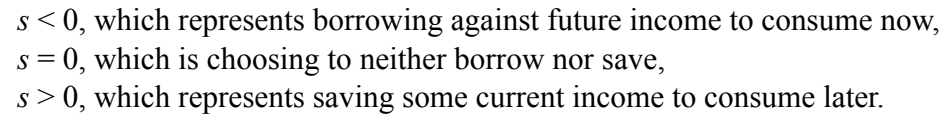

The choice of $s$ is limited by the constraints that saving cannot exceed current income and that the consumer must be able to repay any borrowing. Provided the consumer has the ability to undertake some borrowing and some saving, then $s=0$ is an interior solution, and not a corner solution.

The definition of corner solution in the $3^{\text {rd }}$ edition is intended to capture the basic properties that matter without the need to provide extensive details of context. The definition is:

corner solution In the context of a constrained optimization problem this is a solution that does not change in at least one direction in response to any arbitrarily small perturbation to the gradient of the objective function at the optimum.

This definition can be criticised for not being all that insightful or detailed, but it does have the advantages of being brief and perfectly general. Any improved alternative would need to provide either further mathematical detail (which we wished to avoid) or a convoluted verbal explanation.

The observations made about defining a corner solution take us into a discussion of the appropriate level of mathematics and abstraction in the definitions. This was a central concern in revising the Dictionary. It would have been very easy to produce a formally correct and highly technical dictionary that was of no value to the target audience. In making the revisions it was always in our minds that the typical user was likely to be turning to a dictionary to discover an explanation of an unfamiliar technical concept. It would defeat the purpose of the dictionary if such a user had found a definition written in an equally technical and formal way. This was the dimension in which we needed to explain concepts. Equally, we had to balance this with the need to be correct, and to avoid issues such as those discussed above with corner solution. 
We now return to our Example 3, the definition of concept of equilibrium, to illustrate the different levels of formality that could be used within a definition. The concept of equilibrium is fundamental to economics. Economists conduct their analysis by constructing models and exploring what the models predict. A first step of such an exploration is often to describe what constitutes equilibrium. The philosophy behind this step is that equilibrium is the state of the modelled economic environment that is most likely to emerge from economic activity.

The first step is to interpret equilibrium in the sense of a market equilibrium, and so to focus on supply and demand. Following this approach it is possible to state the conditions for equilibrium with increasing generality and rigor. First, we can have the basic statement that equilibrium occurs when the quantity demanded is equal to the quantity supplied:

(i) Demand $=$ Supply.

Using functional notation for the demand curve and supply curve allows this statement to be recast as

(ii) $\quad D(p)=S(p)$,

where now the quantities demanded and supplied are determined by price. These two statements do not allow the possibility that demand for the good may exceed supply even when the price is zero. We can extend the definition to define equilibrium as a position where

(iii) $\left.\begin{array}{c}p \geq 0 \\ p[S(p)-D(p)]=0,\end{array}\right\}$ with complementary slackness.

The first line states that price cannot be negative, and the second line states that if a good is in excess supply, that is, more is supplied than is demanded, the price of this good should be zero in equilibrium. Finally, the concept can be extended to equilibrium holding simultaneously for many markets and a recognition of the need for price normalisation by using the excess demand function, to write

$$
\text { (iv) } \quad p \in S^{n}, \quad p Z(p)=0 .
$$

The second approach to defining equilibrium is to consider the existence of equilibrium for a game of strategy. The definition of equilibrium then focuses on the choice of strategy by economic agents. An equilibrium set of strategies can be defined in different ways. The simplest is just to state:

(i) An equilibrium is a situation in which no economic agent has an incentive to change strategy.

A more formal definition would bring into account that the strategies are chosen to maximize an objective function.

(ii) An equilibrium is a situation in which the strategies of the $n$ players, $s=$ $\left\{s_{1}, \ldots, s_{n}\right\}$, are such that $s_{i}$ maximizes $V_{i}\left(s_{-i}, s_{i}\right)$ given $s_{-i}$, all $i=1, \ldots, n$.

A final definition is to raise the degree of formality by invoking the best-response mapping, $\rho(s)$ :

(iii) An equilibrium is a situation in which the strategies of the $n$ players, $s=$ $\left\{s_{1}, \ldots, s_{n}\right\}$, satisfy $s=\rho(s)$.

The latter statement would require further definition of the best-response mapping $\rho(s)$ and a discussion of the fixed point argument that is invoked.

Arrow and Debreu used the second approach to demonstrate existence of equilibrium in the first approach. They exploited the fact that there is equivalence between the existence of an equilibrium for a market and the existence of an equilibrium of an appropriately specified game of 
strategy. The different ways in which these ideas are expressed in the $2^{\text {nd }}$ and the $3^{\text {rd }}$ editions can be seen from the alternative definitions.

There are, of course, terms in the $3^{\text {rd }}$ edition for which formal statements with technical details have been given. However, in all such cases a simple descriptive statement is always given first, and some attempt is made to mention the practical use of the concept. This is illustrated in the next example.

\subsection{Example 6}

The final example is illustrative of how it is possible to combine a brief verbal explanation alongside a more formal, and detailed, further description. The definition is made more useful through the inclusion of numerous links to further definitions.

\footnotetext{
expenditure function The minimum cost for a consumer of achieving a given utility level. Consider a consumer choosing the quantities, $x_{1}$ and $x_{2}$, of two goods to minimize expenditure subject to a utility constraint. The cost minimization problem is

$\min _{\left\{x_{1}, x_{2}\right\}} p_{1} x_{1}+p_{2} x_{2}$ subject to $U\left(x_{1}, x_{2}\right) \geq U$.

The solution is described by the two * compensated demand functions

$x_{1}=h_{1}\left(p_{1}, p_{2}, U\right)$ and $x_{2}=h_{2}\left(p_{1}, p_{2}, U\right)$.

Substituting the optimal choices back into the objective gives the minimized level of expenditure as

$E\left(p_{1}, p_{2}, U\right) \equiv p_{1} h_{1}\left(p_{1}, p_{2}, U\right)+p_{2} h_{2}\left(p_{1}, p_{2}, U\right)$.

The function $E\left(p_{1}, p_{2}, U\right)$ is the expenditure function. Shephard's lemma states that $\partial E / \partial p_{i}=h_{i}\left(p_{1}, p_{2}, U\right)$, a result that is useful for calculating the welfare consequences of a price change. See also indirect utility function.
}

\section{Moving online}

There is an ever increasing quantity of information readily available on the internet and the access to the internet is becoming universal. It is perhaps the case already that 100 percent of university students have almost unlimited internet access even in less developed countries. This means that many potential purchasers of the Dictionary find it convenient to look up the meaning of an unfamiliar term online. Academic publishers have recognized this, and have started providing online versions of printed reference sources, just as this was done with academic journals for decades. The Oxford University Press is one of the leaders in this area and even has a separate website for its online reference literature (www.oxfordreference.com). Despite this expertise, the current online version of the Dictionary is no more that the paper version on a different platform.

An online platform offers new opportunities for the Dictionary, which can help address some of the issues that have had to be confronted in producing a paper version. Revision of an online version does not involve the production costs of a paper dictionary, so frequent updates could be made to help eliminate outdated information. Layered information with all cross-references connected by hyperlinks could allow simpler reference to related concepts. In addition to making the use of a dictionary more convenient, layered information would facilitate additional formality and details that could be pursued by the interested user. Links could also be provided to (reputable) external online sources, such as the official websites of international organizations (say, the World Trade Organization in the entry on barriers to trade) or government departments (say, Her Majesty's Revenue and Customs in the entry on tax evasion).

However, as with any resource, exploitation of the technology requires the commitment of the publisher. There may be no funds available for investing in design and support of online sources. The production of a successful online dictionary would require an informed design effort to create a website with enough visual information to make its use easy and transparent, without making visual content so large that time it takes for a web page to download makes it unhelpful and 
frustrates the users. In a traditional publishing house used to dealing with printed material, no expertise may be available to support this effort. The benefits of frequent updating are only gained if the compilers have direct access to the website to make their own revisions. This permission may not be forthcoming if the dictionary is hosted on the publisher's server.

The need to structure linked and layered data will also make the construction of the dictionary more time consuming, but updating can allow this to be distributed over time. Moreover, it could become a continuous process with frequent updating and development provided the compilers could directly post revisions to the hosting web server. The benefits for the users and the popularity of well-designed, user-friendly and timely updated online edition of a dictionary will more than justify the cost invested in its creation.

\section{Conclusions}

In this paper we described our approach to preparing a new edition of the Oxford University Press Dictionary of Economics. The main factor that we had to take into account was that economics has evolved as an academic discipline where research is based on the application of mathematics and statistics. The balance between the level of formality and rigor and the level of accessibility to the intended audience of undergraduate students provides a challenge for the compiler of a dictionary.

To meet this challenge a compiler must be an expert or at least have a good working knowledge of the technical advances in the discipline, and must understand the formalities but also be able to convey highly technical concepts in an accessible language without much loss for the content. Furthermore, in a good dictionary an entry should not be just the statement but also should play an explanatory role. To achieve all of these requires a combination of skills. We hope that the examples in the paper show convincingly that in this regard the revised edition is a step forward. A major limitation of a static printed edition is the time and resources it takes to update and correct the existing entries and to add new entries brought about by the evolution of economics as a discipline and by the new developments in economic and political environment.

An online edition can relax many of the constraints but only if the technology is exploited effectively and efficiently. This seems to be now the direction where the major publishing houses, including Oxford University Press, are moving, to the great benefit of authors and users alike. An online edition represents a significant challenge for the compilers but the benefits would be significant. Possibly the greatest benefit would be the possibility of developing a reference tool that could really meet the needs of a diverse audience rather than the very targeted audience of the print edition.

\section{References}

\subsection{Dictionaries}

Dictionary of Economics $2^{\text {nd }}$ edition $=$ Black, J. 2002: Dictionary of Economics. $2^{\text {nd }}$ edition. Oxford: Oxford University Press.

Dictionary of Economics $3^{\text {rd }}$ edition = Black, J. /Hashimzade, N./Myles, G. D. 2009: Dictionary of Economics. $3^{\text {rd }}$ edition. Oxford: Oxford University Press.

Dictionary of Economics $4^{\text {th }}$ edition $=$ Black, J. /Hashimzade, N./Myles, G. D. 2012: Dictionary of Economics. $4^{\text {th }}$ edition. Oxford: Oxford University Press.

\subsection{Other literature}

Arrow 1951 = Arrow, K. J. 1951: Social Choice and Individual Values. New York: John Wiley.

Arrow/Debreu 1954 = Arrow, K. J./Debreu, G. 1954: Existence of an equilibrium for a competitive economy. In Econometrica 22, 265-290.

Debreu 1959 = Debreu, G. 1959: Theory of Value. New Haven: Yale University Press. 
Hicks $1939=$ Hicks, J. R. 1939: Value and Capital. Oxford: Clarendon Press.

Mas-Collel/Whinston/Green 1995 = Mas-Collel, A./Whinston, M. D./Green, J. R. 1995: Microeconomic Theory. New York: Oxford University Press.

McKenzie 1954 = McKenzie, L. 1954: Equilibrium in Graham's model. In Econometrica 22, 147-161.

Ramsey 1927 = Ramsey, F. 1927: A contribution to the theory of taxation. In Economic Journal 37, 47-61.

Ramsey 1928 = Ramsey, F. 1928: A mathematical theory of saving. In Economic Journal 38, 543-559.

von Neumann/Morgenstern 1944 = von Neumann, J./Morgenstern, O. 1944: Theory of Games and Economic Behavior. Princeton: Princeton University Press. 